\title{
Y A-T-IL UNE ÉLECTIVITÉ CUTANÉE DES TEIGNES ANIMALES ?
}

\author{
Par R. DHAYAGUDE
}

Les champignons des teignes ou dermatophytes sont essentiellement des parasites de la peau et de ses dépendances. Inoculés dans la peau scarifiée de l'homme ou du cobaye, ces champignons produisent des lésions après une période d'incubation de six à huit jours.

L'invasion de la peau peut être démontrée par l'examen microscopique des poils de la région infectée. Quand on examine ces poils dans le chloral-lactophénol, on voit leur envahissement par des filaments et des spores ; dans certains cas, cet envahissement est limité à la surface ; dans d'autres cas, ils pénètrent profondément dans la substance du poil, et, dans un troisième type, on les observe à la fois superficiellement et en profondeur. D'où les trois variétés de lésions ectothrix (1), endothrix (2) et endo-ectothrix (3). L'infection a tendance à guérir spontanément une dizaine de jours après la première apparition du champignon dans la lésion, c'est-à-dire environ 15 jours après la date de l'inoculation.

Il reste à savoir si les dermatophytes possèdent quelque pouvoir pathogène vis-à-vis des autres tissus du corps. Il existe des faits expérimentaux contradictoires, mais on s'accorde en général pour considérer les soi-disant résultats positifs d'infection généralisée comme de simples embolies provoquées par l'introduction massive du champignon dans les différents tissus et organes et que les observateurs ont confondues avec une infection réelle. En effet, on voit toujours en définitive les filaments dégénérer et disparaître sans avoir provoqué de véritables lésions et sans avoir pu se multiplier et envahir les tissus.

Récemment, Brocq-Rousseu, Urbain et Barotte n'ayant obtenu aucun résultat expérimental, macroscopique ou microscopique, en essayant d'infecter les organes de cobayes par des injections intraveineuses massives de cultures de dermatophytes, ont entrepris une intéressante série d'expériences dans lesquelles ils recherchèrent les réactions de la peau scarifiée, après injections intra-veineuses, intrapéritonéales, sous-cutanées et buccales de cultures de dermatophytes. Ils ont trouvé que, sur 19 cobayes infectés par l'une ou l'autre de ces

Annales de Parasitologie, t. IX, $\mathrm{N}^{\circ} 4 .-1^{\text {er }}$ juillet 1931, p. 368-371. 
méthodes et après une période d'incubation de 12 à 15 jours, 13 d'entre eux présentaient des lésions de la peau, dans lesquelles on pouvait trouver les champignons à l'examen microscopique. Se basant sur ces résultats, ils ont conclu que les dermatophytes se localisent toujours à la peau, quel que soit le mode d'infection et, si la peau est lésée, le champignon se développe au lieu de la lésion.

Si nous mettons de côté la voie intra-veineuse, dans laquelle les spores peuvent souiller la petite plaie cutanée, l'infection de la peau, à la suite d'inoculations dans la cavité péritonéale, ou par la voie sous-cutanée ne peut se produire que si les spores et les filaments sont véhiculés par la circulation sanguine ou lymphatique. Mais ceci n'a lieu que si le champignon est pathogène et se reproduit réellement dans les tissus, fait qui se trouverait en contradiction avec ce qui a été observé jusqu'ici. Cependant, très intéressé par les résultats obtenus par ces expérimentateurs, nous avons entrepris des expériences pour déterminer si, oui ou non, la peau pouvait être infectée de cette manière.

Dans nos expériences, pour la raison mentionnée plus haut, nous n'avons pas employé la voie intra-veineuse et nous avons abandonné la voie buccale, à cause des spores qui peuvent passer dans les excréments, contaminer la peau scarifiée du dos de l'animal et donner des résultats faux. Nous nous en sommes done tenus à deux méthodes, dans lesquelles une émulsion concentrée de champignon peut être inoculée sans crainte de contamination ou de transmission mécanique par la circulation. Nous avons choisi pour nos expériences deux types de dermatophytes : (1) Ctenomyces mentagrophytes (Trichophyton gypseum asteroüdes) et (2) Sabouraudites felineus (Microsporum felineum), tous deux connus pour être très virulents et capables de produire une sérieuse infection de la peau scarifiée après un intervalle de 6 à 8 jours. Les cultures employées pour préparer les émulsions avaient été faites sur gélose glycosée ou maltosée ou à l'amidon soluble et étaient âgées de 5 à 10 semaines. La virulence de ces deux souches avait été auparavant vérifiée expérimentalement.

Des émulsions concentrées furent faites en eau de pomme de terre et injectées dans l'abdomen des cobayes à la dose de $2 \mathbf{c m}^{\mathrm{z}}$ par voie sous-cutanée ou intra-péritonéale. Après un intervalle de 2 ou 3 heures, la peau du dos des animaux fut rasée et ensuite scarifiée au moyen de toile d'émeri en prenant toutes les précautions pour éviter la contamination. L'emploi de la toile d'émeri est une excellente méthode de scarification; on obtient une surface couverte de fines coupures et ne saignant pas. 
Nous avons commencé l'examen de la peau rasée à partir du $7^{\text {e }}$ jour et avons pratiqué l'examen microscopique des poils de plusieurs endroits du dos de chaque animal. Cet examen fut continué jusqu'au $17^{\circ}$ jour après l'infection (les limites données par les expérimentateurs étant de 15 jours). Nous n'avons pas trouvé trace d'infection dans la peau ou les poils de la région scarifiée.

\begin{tabular}{|c|c|c|c|c|c|c|}
\hline \multirow{2}{*}{$\begin{array}{c}\text { Mode } \\
\text { D'iNoculation }\end{array}$} & \multicolumn{3}{|c|}{ Ctenomyces mentagrophytes } & \multicolumn{3}{|c|}{ Sabouraudites felineus } \\
\hline & $\begin{array}{c}\text { Nombre } \\
\text { de cobayes }\end{array}$ & Date & Résultats & $\begin{array}{c}\text { Nombre } \\
\text { de cobayes }\end{array}$ & Date & Résultats \\
\hline $\begin{array}{r}\text { Intrapérito- } \\
\text { néale....... }\end{array}$ & 4 & $\begin{array}{c}18-12-30 \\
5-1-31\end{array}$ & négatif & 4 & $\begin{array}{c}18-12-30 \\
5-1-31\end{array}$ & négatif \\
\hline Sous-cutanée. & 2 & $\begin{array}{c}18-12-30 \\
5-1-31\end{array}$ & - & 2 & $\begin{array}{c}18-30-30 \\
5-1-31\end{array}$ & - \\
\hline $\begin{array}{r}\text { Scarification } \\
\text { (les deux sé- } \\
\text { ries de co- } \\
\text { bayes)..... }\end{array}$ & 6 & $\begin{array}{l}20-1-31 \\
27-1-31\end{array}$ & 4 positifs & 6 & $\begin{array}{l}20-1-31 \\
28-1-31\end{array}$ & 6 positifs \\
\hline
\end{tabular}

Nous avons répété nos expériences sur une deuxième série de cobayes avec les mêmes espèces de champignons, exactement de la même manière, et n'avons encore obtenu aucune infection cutanée.

N'ayant obtenu aucune infection de la peau scarifiée par les injections intra-péritonéales et sous-cutanées, nous avons examiné si les précédentes injections avaient produit un effet quelconque sur les animaux. Si le champignon avait été de quelque façon pathogène vis-à-vis de l'animal, celui-ci devrait posséder une immunité ou manifester de l'allergie contre la même espèce de champignon.

Les mêmes cobayes qui avaient servi aux injections furent donc inoculés au bout d'un mois ( 33 jours) sur la partie scarifiée du dos, avec le même champignon. Sur 12 cobayes ainsi inoculés, nous avons obtenu l'infection de 10 d'entre eux. Après une période d'incubation de 5 à 8 jours, les poils rasés repoussèrent presque tous infectés. Les deux résultats négatifs s'expliquent par ce fait que les 2 cobayes avaient le dos couvert de grosses croùtes de sang desséché ; ils étaient dans la même cage et se sont probablement battus 
et mordus. Nous avons remarqué que la présence de sang sur les surfaces scarifiées, empêchait la marche de l'infection. Dans le cas du Sabouraudites felineus, nous avons obtenu une infection avec chaque animal inoculé.

Un animal infecté par scarification et dont les lésions ont spontanément guéri est toujours réfractaire aux réinoculations de la peau. On sait que cet état se prolonge dans le cas des cobayes au delà de 6 à 8 mois. Le fait que, dans nos expériences, nous pouvons obtenir une infection de la peau par scarification, prouve, à notre avis, que la peau des animaux n'a pas été infectée après les injections intra-péritonéales et sous-cutanées.

\section{RÉSUMÉ}

En résumé, de nos expériences, nous sommes arrivés aux conclusions suivantes :

1. - Les dermatophytes n'ont pas de propriété pathogène vis-à-vis des tissus du corps, la peau exceptée.

2. - Après des injections sous-cutanées et intra-péritonéales, il n'est pas possible d'obtenir une localisation du champignon sur la peau scarifiée.

3. - Les précédentes injections sous-cutanées et intra-péritonéales ne produisent pas d'immunité ou d'allergie chez l'animal.

4. - L'animal réagit d'une façon normale après une réinoculation par scarification.

Nous suggérons ici que les résultats positifs obtenus par les expérimentateurs précités doivent être le résultat d'une contamination accidentelle de la peau, soit pendant les expériences, soit après.

\section{BIBLIOGRAPHIE}

Broco-Rousseu, Urbain et Barotte. - Sur l'électivité cutanée des teignes animales, quelle que soit leur voie d'introduction dans l'organisme. $C$. $R$. Soc. de biologie, XCV, 1926, p. 966-968.

Section de mycologie du Laboratoire de parasilologie de la Facultè de Médecine de Paris. 